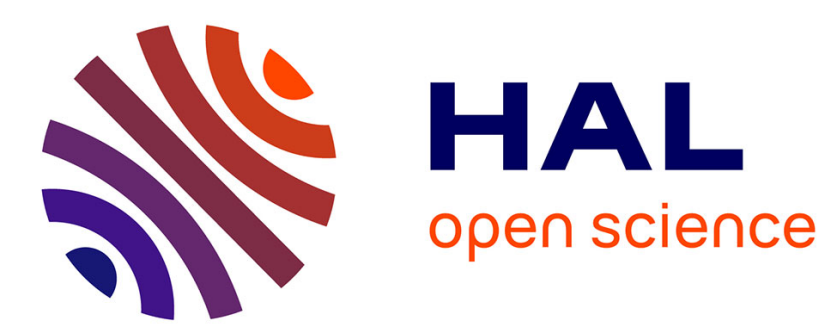

\title{
Mesure du module de cisaillement à faible fréquence au cours du fluage en traction
}

\author{
P. Bourges, J.L. Gacougnolle, J. Woirgard, J. de Fouquet
}

\section{To cite this version:}

P. Bourges, J.L. Gacougnolle, J. Woirgard, J. de Fouquet. Mesure du module de cisaillement à faible fréquence au cours du fluage en traction. Revue de Physique Appliquée, 1978, 13 (1), pp.1-3. 10.1051/rphysap:019780013010100 . jpa-00244416

\section{HAL Id: jpa-00244416 https://hal.science/jpa-00244416}

Submitted on 1 Jan 1978

HAL is a multi-disciplinary open access archive for the deposit and dissemination of scientific research documents, whether they are published or not. The documents may come from teaching and research institutions in France or abroad, or from public or private research centers.
L'archive ouverte pluridisciplinaire HAL, est destinée au dépôt et à la diffusion de documents scientifiques de niveau recherche, publiés ou non, émanant des établissements d'enseignement et de recherche français ou étrangers, des laboratoires publics ou privés. 


\title{
REVUE DE PHYSIQUE APPLIQUÉE
}

Classification

Physics Abstracts

$46.00-36.30 \mathrm{R}$

\section{MESURE DU MODULE DE CISAILLEMENT A FAIBLE FRÉQUENCE AU COURS DU FLUAGE EN TRACTION}

\author{
P. BOURGES, J. L. GACOUGNOLle, J. WOIRGARD et J. DE FOUQUET
}

Laboratoire de Mécanique et de Physique des Matériaux

Université de Poitiers (E. R. A. no 123 du C. N. R. S.)

E. N. S. M. A., rue Guillaume VII, 86034 Poitiers Cedex, France

(Reçu le 24 mars 1977, révisé le 4 octobre 1977, accepté le 12 octobre 1977)

\begin{abstract}
Résumé. - Une méthode permettant la mesure continue du module de cisaillement au cours d'une expérience de fluage en traction est décrite. Cette mesure effectuée au voisinage du $\mathrm{Hz}$, montre des variations importantes du module dynamique en relation avec les différents stades du fluage.
\end{abstract}

Abstract. - A method for measuring the shear modulus continuously during a tension creep test is described. The measurement at about $1 \mathrm{~Hz}$, shows important variations of the dynamic modulus which can be related to the different creep stages.

1. Introduction. - L'évolution structurale des métaux en cours de fluage, en particulier la formation et la modification des arrangements de dislocations a été étudiée par des techniques variées. Parmi celles-ci la microscopie optique [1] et les figures d'attaque [2] permettent d'observer la surface des échantillons, la radiocristallographie [3] et les mesures de densité [4] prennent en compte des effets de volume tandis que la microscopie électronique basse tension [5] permet d'observer la structure des lames d'environ un micron d'épaisseur. Toutes ces méthodes nécessitent la trempe de l'échantillon à un stade donné du fluage.

La microscopie électronique à haute tension [6] permet de suivre le comportement dynamique des dislocations mais en raison des effets de surface ce comportement n'est pas nécessairement celui qui a lieu dans les pièces massives. C'est pourquoi il peut être fructueux de mesurer in situ le module de cisaillement d'une éprouvette en cours de fluage et de comparer ces variations aux variations de vitesse de fluage et de structure.

On sait en effet que si des segments de dislocations sont suffisamment mobiles pour se courber sous l'effet d'une contrainte alternative faible ils entraînent une chute de module de la forme

$$
\frac{\Delta E}{E+\Delta E}=-\frac{N l^{3}}{k}
$$

où

$E:$ module du métal sans dislocation,

$\Delta E$ : écart de module,

$N$ : nombre de segments de dislocations mobiles par unité de volume,

$l:$ longueur libre de ces segments de dislocations,

$K$ : constante égale à 18 si les segments glissent seulement, et à 7 s'ils montent également.

Friedel, Boulanger et Crussard [7] ont utilisé cet effet de module pour étudier le comportement des parois de dislocations dans l'aluminium à haute température.

Le frottement intérieur d'éprouvettes en cours de fluage a été mesuré par G. H. Brook et col. [8] puis par A. Loyer [9] mais l'interprétation de ces mesures est délicate puisqu'au-dessus de $0,5 T_{\mathrm{f}}$ interviennent divers pics de frottement intérieur dont la nature n'est pas clairement établie et qui se superposent aux effets observés.

2. Principe de la mesure. - La période d'oscillation $T$ d'un pendule simple de torsion constitué d'un volant d'inertie $I$ suspendu à un fil de longueur $L$, de diamètre $D$ et de module de cisaillement $G$ est donnée par

$$
T=2 \pi\left(\frac{32 L I}{G \pi D^{4}}\right)^{1 / 2}
$$


On peut aisément déduire les variations de $G$ de la mesure de la période $T$. Dans le cas du fluage du fil de suspension, $L$ et $D$ varient donc et

$$
\frac{\mathrm{d} G}{G}=\frac{\mathrm{d} L}{L}-4 \frac{\mathrm{d} D}{D}-2 \frac{\mathrm{d} T}{T} .
$$

On sait que pour de nombreux métaux la masse volumique $\rho$ diminue au cours du fluage notamment en raison de la création de cavités aux joints de grains [4]. Le nombre de ces cavités devient important à la fin du fluage secondaire et au cours du fluage tertiaire il peut entraîner une chute de densité de l'ordre de $10^{-2}$. Pendant le fluage primaire et le début du fluage secondaire la variation de densité ne dépassant pas $10^{-3}$, nous la négligerons car les variations de module atteignent $10^{-1}$.

Supposant que le volume de l'éprouvette reste constant on a

$$
\frac{G-G_{0}}{G_{0}}=\frac{T_{0}^{2}}{T^{2}} \frac{l^{3}}{l_{0}^{3}}-1 .
$$

Le problème revient donc à réaliser un pendule permettant l'application de contraintes axiales sur une éprouvette sans modifier l'inertie en torsion de l'ensemble du système.

3. Réalisation. - L'équipage d'inertie du pendule (Fig. 1) a été suspendu au fléau d'une balance par un ruban d'acier $F$ de constante de torsion négligeable. Le contrepoids E permet d'annuler toute contrainte axiale sur l'éprouvette. Pour imposer la

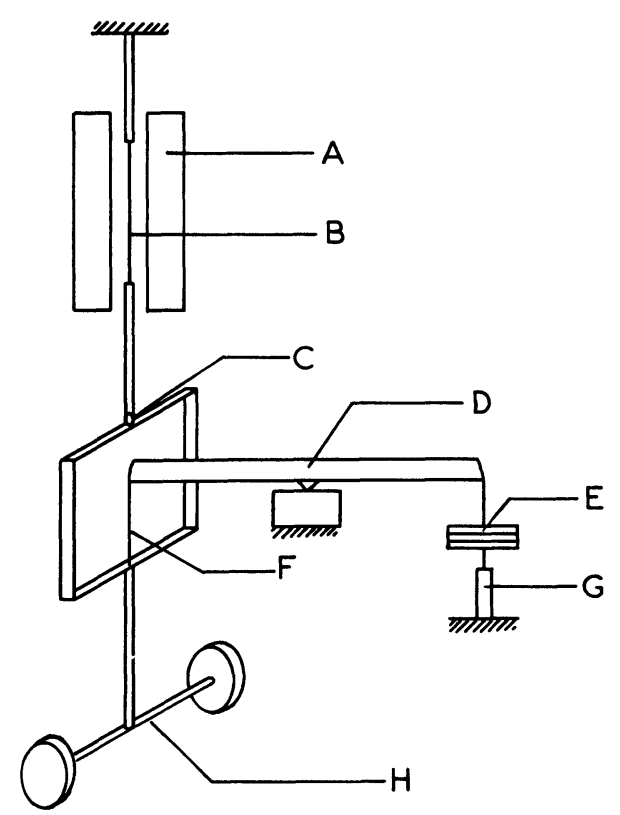

Fig. 1. - Schéma de l'appareil. A : four ; B : éprouvette ; $\mathrm{C}$ : miroir ; D : fléau ; $\mathrm{E}$ : contrepoids ; $\mathrm{F}$ : ruban ; $\mathrm{G}$ : capteur inductif ; $\mathbf{H}$ : équipage inertiel.

[Apparatus diagram. A : furnace ; B : sample ; C : mirror ; $\mathrm{D}$ : beam ; F : counterweight ; F : band ; $\mathrm{G}$ : inductive gauge ; $\mathbf{H}$ : inertia bar.] contrainte de fluage il suffit d'enlever tout ou partie du contrepoids. La contrainte maximale correspond $\mathrm{au}$ cas où l'éprouvette supporte tout l'équipage d'inertie.

L'éprouvette $B$ est un fil de $3 \mathrm{~mm}$ de diamètre et de $80 \mathrm{~mm}$ de longueur fixé à des mordaches lisses, une vis pointeau évitant le glissement. La contrainte peut atteindre $4 \mathrm{MPa}$. Un four à trois enroulements permet d'atteindre dans la version actuelle $950 \mathrm{~K}$ a l'air libre avec un gradient inférieur à $2 \mathrm{~K}$ sur l'éprouvette.

La mesure des allongements est réalisée par un capteur inductif $\mathrm{G}$ placé sous le contrepoids $\mathrm{E}$. Ce dispositif a été choisi afin d'éviter tout frottement sur l'équipage oscillant. La déformation unitaire minimum mesurable est de $2 \times 10^{-4}$. Il a été vérifié que les liaisons mécaniques ne subissent aucun fluage parasite décelable.

La sollicitation en torsion est assurée par deux bobines à noyaux plongeurs. La détection du mouvement est optique. L'amplitude des oscillations est maintenue constante, elle correspond à une déformation de l'éprouvette de $5 \times 10^{-6}$ et à une contrainte de $5 \times 10^{-2} \mathrm{MPa}$. La période est mesurée sur 10 oscillations avec une précision de $5 \times 10^{-4}$ lorsque l'éprouvette ne subit pas la contrainte axiale, après l'application de la contrainte la précision chute à $10^{-2}$. Par ailleurs l'application de la contrainte axiale entraînant une rotation de l'éprouvette, cette rotation a été enregistrée sur un suiveur de spot.

4. Application à l'aluminium. - Les fils d'aluminium de pureté 99,999\% sont recuits in situ 3 heures à $790 \mathrm{~K}$. Les grains obtenus ont un diamètre de l'ordre de grandeur de celui du fil. A l'aide de la microscopie électronique nous avons détecté une polygonisation légère, les sous-grains faisant environ 5 à $7 \mu$ de diamètre. La figure 2 montre la courbe de fluage et la variation de module qui l'accompagne après l'application d'une contrainte de $1,6 \mathrm{MPa}$ à

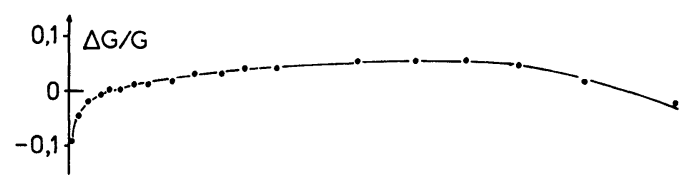

$635 \mathrm{~K} \quad 1,6 \mathrm{MPa}$

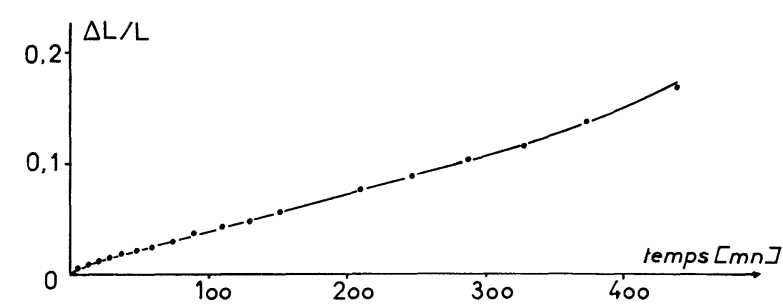

Fig. 2. - Variations de module de cisaillement et d'allongement en fluage à $635 \mathrm{~K}$ sous $1,6 \mathrm{MPa}$.

[Sample torsion during $635 \mathrm{~K}-1.6 \mathrm{MPa}$ creep.] 
$635 \mathrm{~K}$. La première mesure de module s'effectue environ $2 \mathrm{~min}$. après l'application de la charge. On constate que la valeur du module est plus basse d'environ $10 \%$ puis les mesures suivantes font apparaître une augmentation rapide. Il n'est pas actuellement possible de préciser si la chute de module a lieu pendant ou tout de suite après l'application de la contrainte.

L'augmentation rapide du module se déroule au cours du fluage primaire. Pendant le fluage secondaire la croissance est de l'ordre de $5 \%$. Puis une décroissance accompagne le fluage tertiaire, malheureusement il est difficile de faire la part de striction et la part de la structure du métal dans cette décroissance.
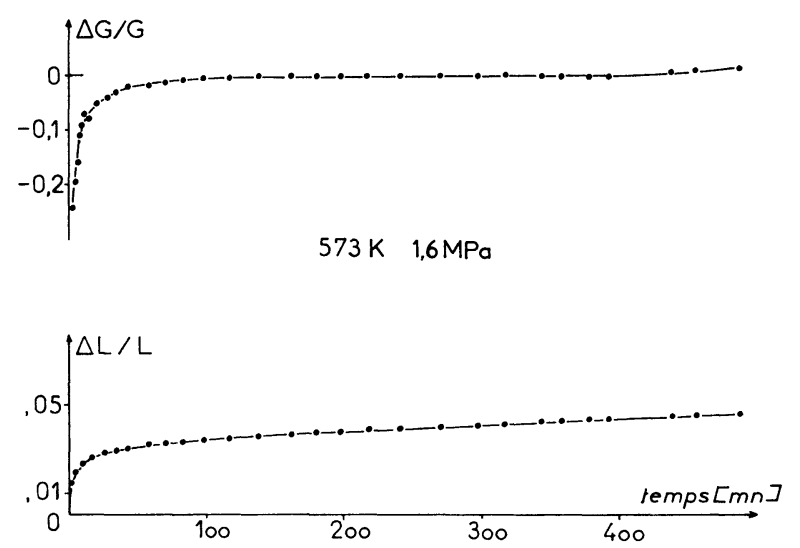

FIG. 3. - Variations de module de cisaillement et d'allongement en fluage à $573 \mathrm{~K}$ sous $1,6 \mathrm{MPa}$.

[Shear modulus and length variations during 635K- $1.6 \mathrm{MPa}$ creep.]
Sous la même contrainte de 1,6 MPa, mais à $573 \mathrm{~K}$ (Fig. 3) la chute initiale mesurée est de $25 \%$. L'augmentation très rapide du module s'arrête au bout de $100 \mathrm{mn}$ un peu avant la fin du fluage primaire à $150 \mathrm{mn}$. Au cours du fluage secondaire la variation de module est très lente, de l'ordre de $1 \%$.

5. Interprétation. - Friedel et col. ont montré qu'au-delà de $500 \mathrm{~K}$ la polygonisation de l'aluminium entraîne une diminution du module d'Young mesuré à une période de $15 \mathrm{~s}$. En effet, la force d'épinglage des segments de dislocations par les impuretés diminue à partir de cette température et les parois de dislocations parallèles se courbent sous la contrainte appliquée. La valeur initiale du module des éprouvettes étudiées ici est déjà plus faible que celle du même métal parfaitement recristallisé. La contrainte de fluage arrache de nombreuses dislocations aux parois et probablement détruit un certain nombre de ces parois suivant un processus similaire à celui décrit par Myshlyaev [10]. Lorsque ces dislocations sont devenues libres la longueur $l$ des segments sensibles à la contrainte de torsion est maximum, la chute de module atteint alors son point le plus bas. Puis les dislocations se multiplient et se rassemblent en parois, la longueur libre moyenne entre 2 nœuds diminue et le module augmente à nouveau pour retrouver une valeur proche de la valeur de départ.

La variation du module au cours des premiers stades de fluage qui est sensible à la structure des dislocations se révèle donc apporter des résultats complémentaires à ceux fournis par la seule courbe d'allongement.

\section{Bibliographie}

[1] Grant, N. J., Chadhuri, A. R., Creep and Recovery Cleveland; (American Society for Metals) p. 284.

[2] Gervais, A. M., Norton, J. T., Grant, N. J., Trans. AIME 197 (1953) 1166.

[3] Dorn, J. E., J. Mech. \& Phys. Solids 3 (1954) 85-116.

[4] Needham, N. G., Wheatley, J. E., Greenwood, G. W., Acta Met. 23 (1975) 23-27.

[5] Orlova, A., CadeK, J., Phil. Mag. 28 (1973) 891.
[6] Myshlyaev, M. M., Caillard, D., Martin, J. L. (1977) Proceed 5th Int. Conf. on High Voltage Microscopy, Kyoto (à paraître).

[7] Friedel, J., Boulanger, C., Crussard, C., Acta Met. 3 (1955) 380.

[8] BrooK, G. H., Sully, A. H., Acta Met. 3 (1955) 460.

[9] Loyer, A., Thèse (D. Sci. A) Montréal 1972.

[10] Myshlyaev, M. M., Proceed. 4th Int. Conf. on the Strength of Met. and Alloys (Nancy) 3 (1976) p. 1037. 